\title{
ESCENARIOS URBANOS EN LA OCUPACIÓN COMERCIAL DE LOS ALREDEDORES DEL CENTRO COMERCIAL LAS MERCEDES EN LA ZONA CÉNTRICA DE CÚCUTA.
}

\section{URBAN SCENARIOS IN THE COMMERCIAL OCCUPATION OF THE PUBLIC SPACE AROUND THE MERCEDES SHOPPING CENTER IN THE DOWNTOWN AREA OF CÚCUTA.}

\author{
Yannette Díaz Umaña ${ }^{1}$ \\ Julio Alfredo Delgado Rojas² \\ Mawency Vergel Ortega ${ }^{3}$
}

Universidad Francisco de Paula Santander.

\section{RESUMEN}

Este artículo se deriva de la investigación sobre la apropiación del espacio público de la zona céntrica de la ciudad de San José de Cúcuta. La investigación se sustentó en un estudio etnográfico que permitió la indagación mediante la entrevista de profundidad y la observación, donde se alcanzó el acercamiento a las historias

1 Magister en Gestión Urbana, Especialista en Docencia Universitaria, Arquitecta, investigadora, docente titular y directora del Programa y Departamento de Arquitectura, Diseño y Urbanismo. Filiación: Universidad Francisco de Paula Santander. Correo electrónico: yannettedu@ufps.edu.co Orcid: https:// orcid.org/0000-0003-4582-1593

2 Magister en Educación Matemática, Arquitecto, Docente. Filiación: Universidad Francisco de Paula Santander. Correo electrónico: julioalfredo@ufps.edu.co Orcid: https://orcid.org/0000-0001-6944-832X

3 Doctora en Educación. Postdoctora en Imaginarios y representaciones sociales. Docente y Directora del Departamento de Matemáticas y Estadística. Filiación: Universidad Francisco de Paula Santander. Correo electrónico: mawency@ufps.edu.co. Orcid: https://orcid.org/0000-00018285-2968 de vida de las personas dedicadas a la actividad informal en el espacio público de los alrededores del centro comercial las Mercedes. Así mismo se examinó el concepto de ocupación comercial y de apropiación del espacio público, para precisar las formas en la que estas personas generan los vínculos con el espacio público, lugar que han escogido para realizar su trabajo informal.

Palabras Claves: espacio público, apropiación, ocupación comercial

\section{ABSTRACT}

This article is derived from research on the appropriation of public space in the downtown area of the city of San José de Cúcuta. The research was based on an ethnographic study that allowed the investigation through in-depth 
interviews and observation, where the approach to the life stories of people engaged in informal activity in the public space around the shopping center was achieved. Mercedes. Likewise, the concept of commercial occupation and appropriation of public space was examined, to specify the ways in which these people generate links with public space, the place they have chosen to carry out their informal work.

\section{KEYWORDS}

public space, appropriation, commercial occupation

\section{INTRODUCCIÓN}

La ubicación fronteriza de la ciudad de Cúcuta, pone de manifiesto una situación perfilada con indicadores que configuran los más altos números de informalidad en Colombia, lo cual se vincula con su naturaleza comercial arraigada en lo histórico (Vergel, Delgado \& Díaz 2020)

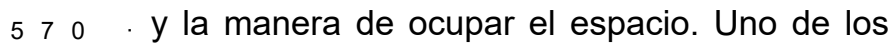
lugares más concurridos y apropiados por parte de los vendedores informales en la ciudad de Cúcuta son los alrededores del centro comercial las Mercedes, de modo que se centra especial interés en esta zona, por lo que la investigación se desarrollada en dos momentos, en primera instancia, una lectura de dicha apropiación en el año 2017 y posteriormente a inicios del 2020.

De este modo el trabajo de campo cumplió un papel importante amparado en Hurtado (2006), quien señala que las fuentes vivas permiten recrear el ambiente natural y constituir un aporte imprescindible con este diseño de campo. Así mismo Páramo (2011) ratifica el concepto de "Trabajo de Campo" como estrategia de la investigación Etnográfica. La población a estudiar fueron los vendedores informales, mayores de edad, quienes cuentan con sus puestos de trabajo situados en los alrededores del centro comercial las Mercedes de la ciudad de Cúcuta. Para Sabino (1992), una población es la concurrencia de todos los casos que concuerdan con una serie de especificaciones, es decir, comparten ciertas características. La técnica usada para la recolección de la información se conoce como "Bola de Nieve" expuesta en Taylor y Bogdan (1987) en Díaz (2014) donde el número total de entrevistas se sabrá al culminar el proceso con la saturación de los datos que suponen la bola de nieve.

Esta técnica está diseñada de tal manera que un informante perteneciente a la población, además de ser entrevistado, recomienda a otro vendedor que a su consideración es representativo dentro de este sistema de ventas informales (Díaz 2014). Este método permitió un mayor acercamiento al propiciar la familiarización con el entrevistado ganando tiempo o protocolos para lograr exitosamente la entrevista de profundidad. Esta estrategia de presentación por terceros, facilitó el proceso, y dio a la investigación un valor agregado en el sentido que son ellos mismos los que se eligen de acuerdo a su representatividad en la población a estudiar. De la misma manera esta estrategia alcanza a facilitar el acceso a la zona por cuanto los grupos al margen de la ley que no ven con buenos ojos los seguimientos y estudios del entorno.

\section{DISCUSIÓN}

Para abordar el tema de la apropiación del espacio público por parte de los vendedores informales, supone examinar el concepto de apropiación para precisar las formas en la que estas personas generan los vínculos con el espacio público, lugar que han escogido para realizar su trabajo informal. La noción de apropiación del lugar según Vidal \& Urrútia (2005), se vincula a un proceso básico del ser humano, donde el dominio de las significaciones del objeto y/ o espacio que es arraigado y se establecen sin importar su verdadera propiedad legal. Para Pol (1996), citando a Chombart de Lauwe, apropiarse de un lugar no es sólo usarlo, sino establecer una conexión, arraigarlo, dejar 
huella, integrarlo en las propias vivencias, y ser protagonista de su transformación. Este autor coincide con Vidal \& Urrutia (2005), en la forma de apropiación, indicando que en la acción que transforma el espacio físico es el proceso primario que él denomina marcaje del territorio. También mencionan el componente simbólico que se produce en la identificación del sujeto o grupo social con el lugar. En esta etapa los procesos afectivos, cognitivos, también tienen participación (Diaz, Parra \& Vergel (2018).

Por su parte Lindon (2009) define los "escenarios urbanos fijos e insertos en el ciclo cotidiano", en la cual uno de sus casos más típicos, es el vendedor informal, para quien esta apropiación del espacio público es el medio para realizar su actividad laboral. Esta autora afirma que para lograr la lógica de empoderamiento realiza, huellas o marcajes físicos en el lugar. También asevera que esta apropiación, puede ir acompañada de una relación de tipo topofílica ${ }^{4}$, aunque este modo de apropiación este implantado solo por un periodo de tiempo limitado.

Otros autores también definen el espacio público como Borja \& Murci (2003), Monnet (2012) que lo cualifican como un lugar social por excelencia. Para Paramo \& Burbano 2011, Veleda Da Silva, 2003 y Mc Dowell, 2000) debido a los patrones sociales preconcebidos lo convierte en un espacio de discriminación y segregación presentando de alguna forma la concepción del espacio público como un objeto mercantilista (Carrión, 2005), y por su función comercial, además de social y cultural (Monnet, 2012). Por otro lado, como expresión de vulnerabilidad económica de un lugar formulado por la ocupación mediante las ventas callejeras

$4 \quad$ Término acuñado por Yori (2007), citando a Tuan como el "Conjunto de relaciones afectivas y de emociones positivas que el ser humano mantiene por un determinado lugar" (p.61), la palabra se desglosa en "topos" como el lugar de convergencia o de encuentro del ser y estar, y philos entendido como "co-apropiante" expresando la idea del encuentro -apropiación del ser, según este autor.
(Veleda Da Silva, 2003), tal como sucede en estos alrededores del centro comercial las Mercedes.

Por su parte Pinho (1986), define la economía informal como actividades ilegales generadoras de renta, no declaradas para efectos fiscales y en consecuencia no reflejados en el producto interno Bruto (PIB) de una región o país, lo cual afecta la economía del estado. Una perspectiva semejante es presentada por Bustamante (2011) como:

"Unidades dedicadas a la producción de bienes o la representación de servicios con la finalidad primordial de crear empleos y generar ingresos para las personas que participan de esta actividad. Estas unidades funcionan típicamente a pequeña escala, con una organización rudimentaria, en la que hay muy poca o ninguna distribución en el trabajo y el capital como factores de producción. Las relaciones de empleoen los casos en que exista- se basan más bien en el empleo ocasional, parentesco o las relaciones personales y sociales y no en acuerdos contractuales que supongan garantías formales." (p. 4).

Ambas concepciones coinciden en subrayar el daño que se genera tanto para el fisco como para las personas la evasión oficial en términos económicos y laborales. Por consiguiente, el término de "vendedores informales" se le conoce como: "Aquellas personas que obtienen un beneficio económico por realizar su actividad comercial en el espacio público sin generarle a la ciudad una retribución económica a cambio." (Conpes, 2012) e incluso el enunciado de Castells (1997) que puntualiza: "[...] aludiendo al uso intensivo del espacio público como un espacio laboral de tiempo completo, más que un espacio del intercambio e intersección entre lugar y flujo, como la teoría lo suele caracterizar" ( $p$. 20). Ilega a ser más reconocido universalmente. 
Esta ocupación mediante las ventas callejeras observadas, está muy relacionada con la historia del espacio público en los últimos cincuenta años en la ciudad de Cúcuta, cuyas características urbanas, presentan altos índices de pobreza a nivel nacional. Al momento, estos indicadores corresponden al $35,5 \%$ por encima de la incidencia nacional del $26 \%$ en el 2017 (DANE, 2017), y 2020 con un incremento al $36,2 \%$ por encima de la incidencia nacional del $27 \%$ según el DANE (2019), ocupando los primeros lugares de pobreza. Entre las causas se contempla una economía frágil basada en el intercambio, una producción industrial escasa, niveles de formación precarios, falta de oportunidades laborales en la formalidad, la situación fronteriza con Venezuela y la alta inmigración, que le otorga a la ciudad, ciertas particularidades que la hacen un territorio especial, frente a las otras ciudades colombianas, tal como afirma la Cámara de Comercio (2000), conexos actualmente con la fuerte inmigración de venezolanos que han conseguido disparar las actividades informales con consecuencias en el detrimento del espacio público.

En cuanto a la informalidad, se señala que la proporción de ocupados informales en las 13 ciudades y áreas metropolitanas fue $46,8 \%$, mientras que para el total de las 23 ciudades y áreas metropolitanas fue $48,0 \%$. De las 23 ciudades y áreas metropolitanas, las que presentaron mayor proporción de informalidad fueron: Cúcuta A.M. (68,0 \%), Sincelejo $(65,2 \%)$ y Santa Marta (62,6\%). Las ciudades con menor proporción de informalidad fueron: Manizales A.M. $(38,5 \%)$, Bogotá D.C. $(41,1 \%)$ y Medellín A.M. $(42,1 \%)$ en el año 2017 y para el año 2019 de las 23 ciudades y áreas metropolitanas, las que presentaron mayor proporción de informalidad fueron: Cúcuta A.M. (71,2\%), Sincelejo (65,3\%) y Santa Marta $(63,6 \%)$. Las ciudades con menor proporción de informalidad fueron: Manizales A.M. $(38,2 \%)$, Bogotá D.C. $(39,4 \%)$ y Medellín A.M. (40,8\%), según reportes del DANE 2017 y 2019. (Ver figura 1)

1. Figura 1

\section{Informalidad en Colombia año 2017 y 2020,} principales indicadores.

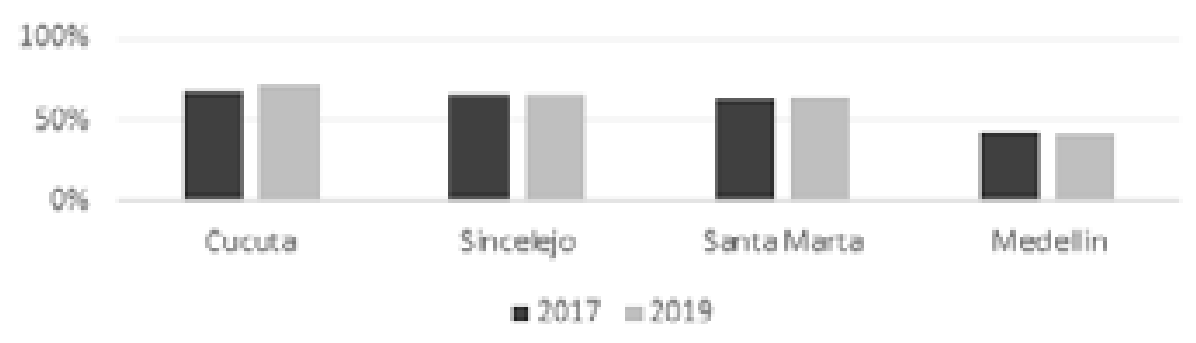

Figura 1. Elaboración propia según fuentes DANE 2017y 2020 
En las observaciones y análisis se pudo notar con la técnica de recolección de información "bola de nieve" puesta en marcha para esta investigación, que de 47 casos registrados, antes de llegar a la repetición y saturación de la datos obtenidos, el $68 \%$ en el año 2017 y el $79 \%$ del 2020 corresponden a mujeres. También se pudo determinar que las personas entrevistadas en el 2017 , vendían productos perecederos en un $90 \%$, distribuidos en frutas $35 \%$, verduras $40 \%$, cárnicos $10 \%$, hierbas y plantas $10 \%$ y otros productos $10 \%$. en contraposición para el 2020 esta distribución varia aumentando la venta de frutas a un $40 \%$, cárnicos $12 \%$ y otros productos $14 \%$. Con respecto a la nacionalidad de los vendedores informales, se pudo advertir que en el 2017 un $40 \%$ eran de origen cucuteño, $32 \%$ de origen venezolano y otras regiones $28 \%$. Para el 2020 es notable el aumento de vendedores venezolanos, pues quienes respondieron la entrevista configuran una población de: $25 \%$ cucuteños, $55 \%$ de origen venezolano y $20 \%$ provenientes de otras regiones.

Igualmente se pudo notar que los más de 250 puestos de venta informal en esta zona de la ciudad, después del desalojo de 2018 seis meses después de la primera lectura, retornaron al lugar, de manera que para la recolección de datos del 2020, la técnica de la bola de nieve, perfilo de nuevo a 30 casos de los 47 , como cabezas visibles de la población de vendedores informales, 17 casos fueron líderes emergentes. Se mantuvo el sistema de cooperativismo, asociación e identificación de líderes entre los vendedores, características que persistieron a pesar del desalojo. El nivel de escolaridad aumento, del $15 \%$ en la lectura del 2017 , que había culminado los estudios de básica y media, a un $25 \%$ en el 2020 destacando que este indicador es mayor en la población venezolana. No obstante se ha convertido en un problema que trasciende de lo económico, social y cultural para decantar en un déficit cuantitativo y cualitativo del espacio público, Tal como el Conpes 3718 del 2012 lo enuncia en las problemáticas del espacio público.

Estas estadísticas refrendan la teoría Veleda Da Silva (2003) y Perrons (2000), al revelar la vulnerabilidad de la mujer en los aspectos laborales, la relación de la pobreza, pocas oportunidades laborales y académicas en la ocupación del espacio público. Los anteriores razonamientos presentan las dos facetas del comercio informal, uno sustentado en la deserción económica y social que corroe la economía al existir evasión de impuestos y, al desproveer al trabajador de cualquier amparo prestacional y por el otro la poca confianza en el gobierno para asumir responsabilidades en situaciones de recesión, así como también en la cultura a la informalidad.

Dicho de otro modo, la economía informal representa la cotidianidad de muchas personas, que en procura de su subsistencia buscan alternativas en la informalidad. El permitir y estimular la actividad comercial en las calles, ya sea desde el propio régimen estatal, o desde la misma formación consumista y transgresora de las normas. Es una acción con consecuencias, ya que altera la economía y la cultura de una sociedad, denotándolo en el espacio público.

El aporte de Veleda Da Silva (2003) en este fenómeno, lo hace desde una descripción cualitativa, basada en diferentes autores, sobre las características del comercio callejero en disímiles escenarios, perfectamente equiparable en algunos aspectos, al comercio informal en las calles de Cúcuta. Esta autora relaciona el fenómeno con los tres enfoques que dan origen a la informalidad: el estructuralista, el neoliberal y el basado en nuevas formas de organizaciones. El primero se explica desde la insuficiencia en la generación de empleos y el alto crecimiento de la fuerza laboral, el segundo 
desde la inflexible regulación del estado y el tercero correspondiente a consecuencias de la crisis económica de los países desarrollados.

\section{CONCLUSIONES}

En ultimas se encontraron problemáticas asociadas a la venta informal, como una mayoría poblacional del género femenino con ello, el compromiso forzoso de la vida laboral y familiar en el espacio público, predominancia de ventas informales de comestible perecederos $\mathrm{y}$ aumento de los vendedores de origen venezolano. Entre otros fenómenos, se observó la modificación del espacio público mediante una apropiación territorial asociaciada al usufructuó del espacio público, integración de una actividad lucrativa, como parte de la cultura de sus antepasados, relacionado con la manera con que las vendedores informales han adquirido su saber hacer que se ha heredado socialmente en el tiempo, y/o un saber hacer determinado por las consecuencia de la inmigración masiva de venezolanos.

Se determina que el proceso de apropiación por parte de los vendedores informales de esta zona, ha presentado signos reconocibles de arraigo al espacio público. Las conductas influidas por la relación fronteriza se evidencian en los procesos cognitivos, afectivos y simbólicos, que representan la binacionalidad en las afueras del centro comercial las Mercedes. El resultado que se presenta es el déficit cualitativo y cuantitativo del espacio público, con pocas posibilidades del goce de este espacio, en la medida que es imposible circular y permanecer. Es de considerarse que toda intervención de este espacio público, está directamente articulada con el sistema económico, además de lo social y cultural, de tal manera que requiere esfuerzos de un equipo coordinado de la Administración Municipal, que sepa liderar la gestión, la planeación y ejecución de acciones pertinentes a la recuperación del espacio público, en sintonía con la comunidad en general, en especial con el grupo de vendedores informales.

\section{REFERENCIAS BIBLIOGRÁFICAS}

Borja J, Muxí Z (2001) El espacio público: ciudad y ciudadanía. Barcelona. Ed Electra Diputación de Barcelona.

Burbano, A. y Páramo, P. (2007). La experiencia de la mujer en el espacio público a Partir de lo rol social. Pre-til, 5 (13), 8-28. Año 5. Http://iaps. Scix.net/data/ contrib/att/bfe7.content.0 .

Bustamante, J. (2011). Los Retos de la Economía Informal en Colombia. Ministerio de Hacienda y Crédito Público- Dirección General de Política Macroeconómica .Centro de Estudios Fiscales. N9 Bogotá. http://www.minhacienda.gov. co/MinHacienda/haciendapublica/ CentroEstudios/publicaciones/notas

Carrión, F (2005). Espacio Público: punto de partida para la alteridad. Facultad latinoamericana de ciencias sociales, Flacso. http://www. bogotacapitaliberoamericanadelacultura. gov.co/.../conferencia\%20

Castell, M. (1995). La ciudad informacional: Tecnologías de la información, restructuración económica y el proceso urbano-regional. Madrid: Alianza Editorial. http://msvelando.com.ar/ tecinfo/documentos/castells ciudad informal.pdf

Conpes 3718 (2012). Política Nacional de Espacio Público. Departamento Nacional de Planeación. Bogotá. https:// www.dnp.gov.co/LinkClick.aspx?filetick et=DnfcXXIwbFM\%3D\&tabid=1475

DANE, (2019). Boletín técnico. Gran Encuesta Integrada de Hogares (GEIH). https://www. dane.gov.co/files/investigaciones/boletines/ 
ech/ech informalidad/bol ech informalidad ago19 oct19.pdf

DANE, (2018). Boletín técnico Gran Encuesta Integrada de Hogares (GEIH). https://www.incp. org.co/wp-content/uploads/2018/10/boletintecnico-dane1.pdf

Díaz, Y. (2014). Mujeres, apropiación conflictos Mujeres, y espacio público en el de la ciudad de Cúcuta. Papeles de Coyuntura N 38 . P.117-124. http://repository.unipiloto.edu.co/ handle/20.500.12277/8294

Diaz-Umaña, Y. ., Parra-López, H. M. ., \& VergelOrtega, M. . (2018). Espacio público: indicadores afectados por ventas informales en la ciudad de Cúcuta. Eco Matemático, 9(1), 43-50. https://doi. org/10.22463/17948231.1725

Guergil, M. (1988). Algunos alcances sobre la definición del sector informal. Revista de

Hurtado, J. (2006). El proyecto de investigación: metodología de la investigación holística. Cuarta Edición. Bogotá, Colombia: Quirón.

Lindon, A. (2009). La construcción socioespacial de la ciudad: el sujeto cuerpo y el sujeto sentimiento. Cuerpos, emociones, y sociedad. Córdoba; N1, año 1, pp. 0620. http://www.relaces.com.ar/index. php/relaces/article/view/4/4

Martínez, D.; Tokman, V. E. (1999). Efectos de las reformas laborales: entre el empleo y la desprotección. En: Tokman, V. E.; Martínez, D. Flexibilización en el margen: la reforma del contrato de trabajo. Lima: OIT. Capítulo I.

McDowell, L. (2000). Género, Identidad y Lugar: Un Estudio de Las Geografías Feministas. Madrid: Editorial Cátedra.

Monnet J. (2012). El Espacio Público definido por sus usos. Una Propuesta Teórica. http:// virtual.unipiloto.edu.co/file.php/966/ Lecturas_de_la_catedra/usos_del espacio_publico-Monnet20121221_1. pdf

Páramo, P. (2011). La investigación de las ciencias sociales: estrategias de investigación. Universidad Piloto de Colombia.

Páramo, P. \& Burbano, A. (2011). Género y espacialidad: análisis de factores que condicionan la equidad en el espacio público urbano. Universitas Psychologica V. 10 No. 1 enero-abril 2011 p.p. $61-70$

Perrons, D. (2000). Flexible working and equal opportunities in the United Kingdon: a case study from retail in: environment and planning A. Vol 32 pp1719-1734.

Pinho, D. (1986). Economía informal, tecnología apropiada e associativismo. IPEA. Sau Paulo: Serie Relatorios de pesquisa.

Pol, E. (1996) La apropiación del espacio. En L.Iñiguez y E.Pol (Coord) Cognición, representación y apropiación del espacio. Barcelona, Publicacions Universitat de Barcelona, Monografies Psico/ Socio/Ambientals $\mathrm{n}^{\circ} 9 \mathrm{http}: / /$ www.ub.edu/escult/doctorat/ html/lecturas/apropia.pdf

Portes, A. \& Haller, W. (2004). La economía informal. División de Desarrollo Social Serie, Políticas Sociales. CEPAL. http://www.cepal.cl/publicaciones/ xml/5/20845/sps100_cl2218.pdf 
Taylor, S. \& Bogdan, R. (1987).Introducción a los métodos cualitativos de investigación. Barcelona: Paidós

Tokman, V. (2001). De la informalidad a la modernidad. Oficina Internacional del Trabajo, Santiago. http://www.oitchile. cl/pdf/publicaciones/emp/emp005.pdf

Vergel Ortega, M.., Delgado Rojas, J. A.., \& Díaz Umaña, Y. (2020) a. Dimensión histórica de la red ferroviaria, su impacto económico y urbano en San José de Cúcuta. Revista Boletín Redipe, 9(6), 183-191. https://doi.org/10.36260/rbr. v9i6.1011

Veleda da Silva, S. (2001). Trabajo informal en América Latina: el comercio callejero. biblio3w, revista bibliográfica de geografía y ciencias sociales universidad de Barcelona. : http://www. ub.edu/geocrit/b3w-317.htm

Veleda Da Silva, S. (2003). Trabajo informal, género y cultura: el comercio callejero e informal en el Sur de Brasil. Universidad Autónoma de Barcelona, Facultad de Filosofía y letras, Departamento de Geografía. Material magnético.

Vidal, T. \& Urrutia, E. (2005). La apropiación del espacio: una propuesta teórica para comprender la vinculación entre las personas y los lugares. Anuario de Psicología, vol. 36, $n^{\circ} 3,281-297$. Facultad de Psicología. Universitat de Barcelona. http://www.raco.cat/ index.php/AnuarioPsicologia/article/ viewFile/61819/81003\&a=bi\&

Yori, C. (2007). Topofilia o la Dimensión Poética del habitar, segunda edición. Santafé de Bogotá: Editorial Pontificia Universidad Javeriana 